\title{
NEWS: the 2019 Metabolomics publication awards
}

\section{Royston Goodacre ${ }^{1}$}

๑) Springer Science+Business Media, LLC, part of Springer Nature 2019

Every journal publishes papers and reviews that they are very proud of, and Metabolomics is no different. In order to recognize the excellent research published in this journal the following prizes will be awarded to the most downloaded original articles and reviews published in the previous year:

- Best Paper Award-for the Original Article with the highest total number of downloads during the previous year. The first author of the winning paper receives a complimentary ebook from Springer (value 200€), a front cover image in the Metabolomics journal, and the paper will be made freely available for 30 days.

- The runner-up paper will also be made freely available for 30 days.

- Best Review Award-for the Review Article with the highest total number of downloads during the previous year. The first author of the winning paper receives complimentary e-books from Springer (value 200€), a front cover image in the Metabolomics journal, and the paper will be made freely available for 30 days.

- The runner-up review will also be made freely available for 30 days.

In order for a paper to be eligible for these awards it must be either a primary research paper (which may include best practice or standards articles, as well as letters and short communications) or a review article (which includes white papers) that was published in its final form in 2018. All awards will be based on popularity via downloads.
Congratulations to the following 2019 winners!!!

\section{Best Paper Award published in 2018}

- Winner-Pan, D., Lindau, C., Lagies, S., Wiedemann, N. \& Kammerer, B. (2018) Metabolic profiling of isolated mitochondria and cytoplasm reveals compartmentspecific metabolic responses. Metabolomics 14: 59. https ://doi.org/10.1007/s11306-018-1352-x

- Runner up-Catala, A., Culp-Hill, R., Nemkov, T. \& D'alessandro, A. (2018) Quantitative metabolomics comparison of traditional blood draws and TAP capillary blood collection. Metabolomics 14: 100. https://doi. org/10.1007/s11306-018-1395-z

\section{Best Review Award published in 2018}

- Winner-Broadhurst, D., Goodacre, R., Reinke, S.N., Kuligowski, J., Wilson, I., Lewis, M. \& Dunn, W.B. (2018) Guidelines and considerations for the use of system suitability and quality control samples in mass spectrometry assays applied in untargeted clinical metabolomic studies. Metabolomics 14: 72. https://doi. org/10.1007/s11306-018-1367-3

- Runner up-Rosato, A., Tenori, L., Cascante, M., De Atauri Carulla, P.R., Martins dos Santos, V.A.P. \& Saccenti, E. (2018) From correlation to causation: analysis of metabolomics data using systems biology approaches. Metabolomics 14: 37. https://doi.org/10.1007/s1130 6-018-1335-y

Royston Goodacre

roy.goodacre@liverpool.ac.uk

Department of Biochemistry, Institute of Integrative Biology, University of Liverpool, Biosciences Building, Crown Street, Liverpool L69 7ZB, UK 